\title{
Association of zinc administration with growth suppression of intracranial aneurysms via induction of $\mathrm{A20}$
}

\author{
Kosuke Hayashi, MD, ${ }^{1,2}$ Hiroharu Kataoka, MD, PhD, ${ }^{1}$ Manabu Minami, MD, PhD, ${ }^{2}$ \\ Taichi Ikedo, MD, PhD, ${ }^{1,2}$ Takeshi Miyata, MD,, Kampei Shimizu, MD, ${ }^{1}$ Manabu Nagata, MD,, \\ Tao Yang, MD, ${ }^{1,2}$ Yu Yamamoto, MD, ${ }^{1,2}$ Masayuki Yokode, MD, PhD, ${ }^{2}$ and \\ Susumu Miyamoto, MD, PhD'
}

${ }^{1}$ Department of Neurosurgery and ${ }^{2}$ Department of Clinical Innovative Medicine, Kyoto University Graduate School of Medicine, Kyoto, Japan

\begin{abstract}
OBJECTIVE Zinc is an essential micronutrient with multiple biological effects, including antiinflammation. Previously, the authors demonstrated that the pathogenesis of intracranial aneurysms (IAs) is strongly related to chronic inflammation. In this study, the authors investigated whether administration of zinc inhibits the growth of IAs in a rat model.
\end{abstract}

METHODS The authors analyzed surgically induced IAs in Sprague-Dawley male rats, which were subsequently treated with intraperitoneal injections of zinc sulfate heptahydrate $\left(\mathrm{ZnSO}_{4} ; 3 \mathrm{mg} / \mathrm{kg} / \mathrm{day}\right)$ or vehicle for 4 weeks.

RESULTS Size and wall thickness ratios of experimentally induced IAs were assessed in both treatment groups after induction and in a control group. The effects of zinc administration in IAs were examined by immunohistochemistry and Western blotting. Zinc administration significantly suppressed aneurysm size and also preserved the internal elastic lumen. Administration of zinc significantly attenuated infiltration of macrophages into IAs.

CONCLUSIONS Zinc treatment significantly increased expression of the antiinflammatory signaling protein A20, an inhibitor of the nuclear factor $\kappa B(N F-\kappa B)$ pathway, in rat IAs. Zinc administration may prevent the growth of rat IAs by inducing $\mathrm{A} 20$-attributed inactivation of $\mathrm{NF}-\kappa \mathrm{B}$ signaling.

https://thejns.org/doi/abs/10.3171/2020.1.JNS192047

KEYWORDS zinc; A20; intracranial aneurysms; nuclear factor $\kappa \mathrm{B}$; vascular disorders

I NTRACRANIAL aneurysms (IAs) are a relatively common lesion, with an incidence of $1 \%-5 \%$ in large autopsy studies. ${ }^{1}$ IAs are the main cause of subarachnoid hemorrhage (SAH), which is associated with an excessive rate of mortality and disability in survivors. The age of onset of SAH is like to be younger than that of cerebral infarction, meaning that the severe impairment of healthy life expectancy and quality of life in SAH survivors is one of the most important medical issues. ${ }^{2}$ Despite the clinical and public health importance of IAs, the mechanisms of initiation, progression, and rupture are still largely un- clear. $^{3}$ As a result, no effective medical treatment for IAs yet exists, although surgical clipping or coiling (which risks complication) is attempted in some cases. ${ }^{4}$ Therefore, to reduce the burden of this condition on public health, it is necessary to develop novel therapies for it.

IAs are associated with nuclear factor $\kappa \mathrm{B}(\mathrm{NF}-\kappa \mathrm{B})-\mathrm{de}-$ pendent inflammation in cerebral arterial walls. ${ }^{3} \mathrm{NF}-\kappa \mathrm{B}$ is a crucial transcriptional factor associated with many biological processes, including immune responses and inflammatory responses. Consistent with this role, $\mathrm{NF}-\kappa \mathrm{B}$ is activated by a huge array of stimuli containing proinflam-

ABBREVIATIONS AAA = abdominal aortic aneurysm; $A C A=$ anterior cerebral artery; $A S T=$ aspartate aminotransferase; EvG = Elastica van Gieson; $I A=$ intracranial aneurysm; $\mathrm{NF}-\kappa \mathrm{B}=$ nuclear factor $\kappa \mathrm{B} ; \mathrm{OA}=$ olfactory artery; $\mathrm{ROS}=$ reactive oxygen species; $\mathrm{SAH}=$ subarachnoid hemorrhage; $\mathrm{ZnF}=$ zinc finger; $\mathrm{ZnSO} \mathrm{O}_{4}=$ zinc sulfate heptahydrate.

SUBMITTED July 30, 2019. ACCEPTED January 20, 2020.

INCLUDE WHEN CITING Published online March 27, 2020; DOI: 10.3171/2020.1.JNS192047. 
matory cytokines and by oxidative stress. ${ }^{5}$ Consequently, $\mathrm{NF}-\mathrm{KB}$ signaling is implicated in a wide variety of disorders, including autoimmune diseases and chronic inflammation.

Zinc has antiinflammatory activities, and zinc supplementation can decrease the activation of NF- $\kappa \mathrm{B} .{ }^{6}$ As a negative regulator of NF- $\kappa \mathrm{B}$, zinc induces the expression of A20, a pleiotropic cytoplasmic protein that is widely accepted as an inactivating factor for the NF- $\mathrm{KB}$ pathway. 5,7 A20, which contains 7 zinc fingers ( $\mathrm{ZnFs}$ ), requires zinc ions to stabilize its overall architecture. ${ }^{8}$ Zinc also has antioxidant activities. ${ }^{5}$ In particular, zinc contributes to the cell's antioxidant defense against reactive oxygen species (ROS), and ROS accumulation and scavenging pivotally participate in the growth of IAs..$^{9,10}$ Moreover, zinc deficiency is known to elicit vascular inflammation related to $\mathrm{NF}-\mathrm{\kappa B}$ signaling ${ }^{10}$ and is thus associated with the formation of IAs mediated by NF- $\kappa \mathrm{B}$ activation. ${ }^{2}$

In this investigation, we focused on the relationship between IAs and A20. Because zinc represents a potentially effective therapeutic agent against IAs, we sought to determine whether administration of zinc prevents the growth of experimental IAs. Here, we describe new findings that shed light on the zinc-A20 axis, which plays a key role in controlling IA growth via mitigation of NF- $\mathrm{KB}$ activation.

\section{Methods}

\section{Induction of Experimental IAs in Rats}

Experiments were approved by the institutional animal care and use committee and conducted according to the national and institutional guidelines for the welfare and management of animals. Seven-week-old Sprague-Dawley male rats (SLC) were randomly separated into 3 groups: control (without any treatment), vehicle treated, and zinc treated. Experimental IAs in rats were induced as previously described. ${ }^{11}$ Briefly, under anesthesia induced with pentobarbital (Kyoritsu Seisaku Corporation; $50 \mathrm{mg} / \mathrm{kg}$ intraperitoneal injection), the left common carotid as well as the left renal arteries were concurrently ligated with 10-0 nylon. Thereafter, rats were fed a high-salt diet containing 8\% sodium chloride (Oriental Yeast) and $0.12 \%$ $\beta$-aminopropionitrile (Tokyo Chemical). After the surgery, the zinc-administered group was given an intraperitoneal injection of zinc sulfate heptahydrate $\left(\mathrm{ZnSO}_{4}\right.$; Wako Pure Chemical Industries) daily at a dose of $3 \mathrm{mg} / \mathrm{kg} /$ day for 4 weeks, whereas the vehicle group was administered with an equal amount of phosphate-buffered saline (Takara Bio). The dosage and route of zinc administration were determined based on previous studies. ${ }^{12,13}$ Four weeks after the operation, these rats were anesthetized with pentobarbital (50 mg/kg injected intraperitoneally), and their brains were fixed with $4 \%$ paraformaldehyde (Wako Pure Chemical Industries) overnight and dipped in 30\% sucrose (Wako Pure Chemical Industries) for 1 day. The bifurcation of the right anterior cerebral artery (ACA) and the right olfactory artery (OA) was stripped, and the sample was frozen in an optimum cutting temperature compound (Sakura Finetek Japan) at $-80^{\circ} \mathrm{C}$. Sections were sliced at a thickness of $5 \mu \mathrm{m}$ using a Cryostat CM 1860 (Leica) and observed under a light microscope after Elastica van
Gieson (EvG; Muto Pure Chemicals) staining. To examine pathological changes in IAs, we measured aneurysm size and wall thickness ratio. Aneurysm size was calculated using BZ software (Keyence) as the mean of the IA maximal longitudinal diameter and maximal transverse diameter of IAs. The wall thickness ratio of an IA refers to the ratio of the minimum width of the aneurysmal wall to the average thickness of the normal arterial wall. Blood pressure was measured by the tail cuff method using a BP-98A blood pressure meter (Softron) 3 times in each rat without anesthesia. Four weeks after the operation, rats were killed under general anesthesia. In total, 165 rats (control group, $\mathrm{n}=45$; vehicle group, $\mathrm{n}=60$; zinc administration group, $n=60$ ) were used in this study. Serum concentrations of zinc, copper, aspartate aminotransferase (AST), and creatinine were measured by Falco Biosystems.

\section{Antibodies}

Primary antibodies were mouse monoclonal antibody against A20 (Santa Cruz Biotechnology), rabbit polyclonal antibodies against Iba-1 (ionized calcium binding adaptor molecule 1) (Wako Pure Chemical Industries), phos-

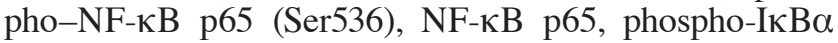
(Ser32/36), and IкB $\alpha$ (Cell Signaling Technology). Rabbit polyclonal $\beta$-actin antibody (Cell Signaling Technology) was used as an internal control.

\section{Immunohistochemical Staining}

After blocking with $2 \%$ bovine serum albumin (SigmaAldrich), 5- $\mu$ m-thick sections were incubated with the primary antibodies for 2 hours at room temperature, followed by incubation for 1 hour at room temperature with Alexa Fluor 488- or 594-conjugated secondary antibodies (Thermo Fisher Scientific). The glass slides covered with Fluoroshield with DAPI (Sigma-Aldrich) were observed on a BZ-9000 fluorescence microscope (Olympus).

\section{Western Blotting}

Four weeks after aneurysm induction, the 2 whole Willis rings were homogenized and dissolved in radioimmunoprecipitation assay (RIPA) buffer (Wako Pure Chemical Industries) containing 10\% protease inhibitor cocktail tablets (Roche) and 1\% PhosSTOP (Roche). After the protein was denatured in loading buffer, supernatants were loaded for Western blotting with the primary anti-

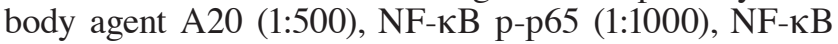

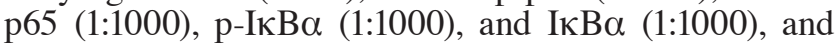
$\beta$-actin (1:1000) was used as an internal control. Experiments were performed in triplicates. The intensity of each band was quantified with ImageJ software (NIH, https:// imagej.net/Citing).

\section{Statistical Analysis}

The results are represented as the means \pm standard deviations (SDs). The mean differences in continuous measurements were assessed with the Mann-Whitney U-test, while differences among multiple groups were assessed with the Kruskal-Wallis test and checked using the Scheffe test. Any $\mathrm{p}$ values less than 0.05 were regarded as statistically significant. 
A Blood Pressure (systolic)
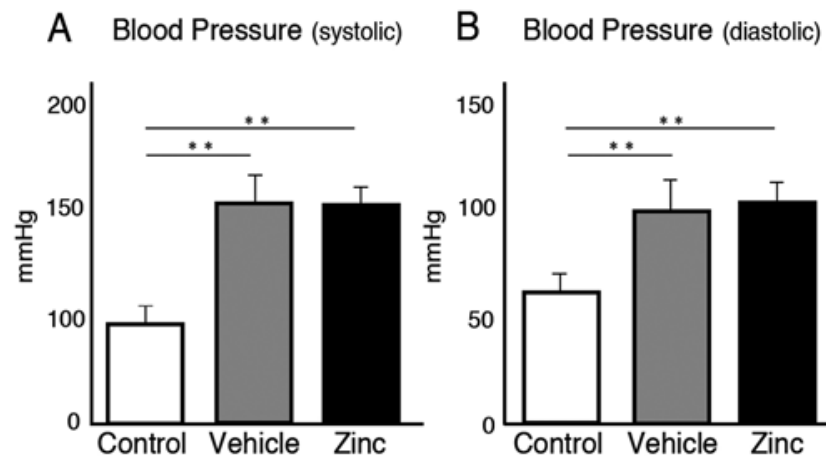

C Heart Rate

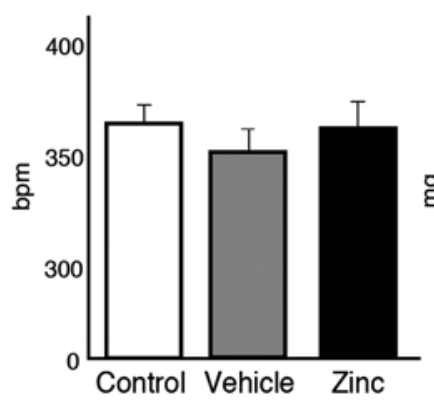

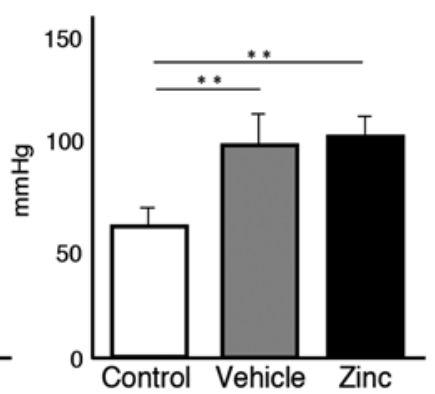

D Body Weight

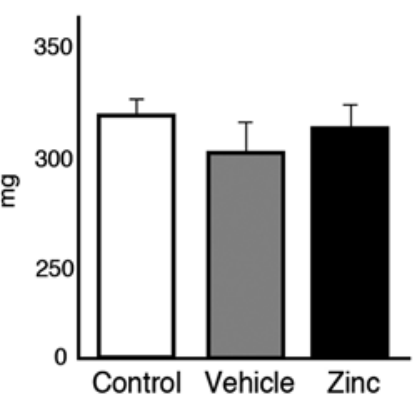

FIG. 1. Systolic (A) and diastolic (B) blood pressure, heart rate (C), and body weight (D) data in rats 4 weeks after aneurysm induction and in the control group were prepared for evaluation. Data were statistically analyzed using the Kruskal-Wallis test. Results are presented as means \pm SDs for $n=9$ per group, ${ }^{* *} p<0.01$. The total number of animals used was 9 in each group.

\section{Results}

\section{Zinc Supplementation Inhibited the Growth of IAs}

Four weeks after aneurysm induction, before the rats were killed, we collected data for comparison of the systolic blood pressure, diastolic blood pressure, heart rate, body weight, change in body weight, and blood values between the control group and the vehicle- and zinc-administered groups. The blood pressure values (systolic/ diastolic) of the zinc- and vehicle-administered groups were higher than those of the control group (zinc, $150.7 \pm$ 9.7/100.9 \pm 9.4 ; vehicle, $150.8 \pm 8.4 / 100.3 \pm 6.4$; and control, $98.1 \pm 4.3 / 61.5 \pm 4.7 \mathrm{~mm} \mathrm{Hg}, \mathrm{p}<0.01$; Fig. $1 \mathrm{~A}$ and B). Heart rate (zinc, $358.7 \pm 19.2$; vehicle, $347.8 \pm 11.7$; and control, $363.2 \pm 17.2 \mathrm{bpm}$ ) and body weight (zinc, $307.2 \pm$ 15.4; vehicle, $299.5 \pm 14.4$; and control, $327.2 \pm 10.7 \mathrm{mg}$ ) did not differ significantly between the 3 groups (Fig. 1C and D). The serum concentration of zinc was significantly higher in the zinc-administered group than in the control and vehicle groups (zinc, $144.5 \pm 1.9$; vehicle, $9.48 \pm 4.2$; and control, $9.38 \pm 4.7 \mu \mathrm{g} / \mathrm{dL}, \mathrm{p}<0.01$; Fig. $2 \mathrm{~A}$ ). In general, the serum concentrations of zinc and copper are closely regulated by compensatory mechanisms; however, in our study, the serum concentration of copper did not differ significantly between the 3 groups (zinc, $173 \pm 4.7$; vehicle, $182.4 \pm 6.6$; and control, $180.1 \pm 6.1 \mu \mathrm{g} / \mathrm{dL}$; Fig. $2 \mathrm{~B}$ ). Serum levels of AST (zinc, $130.1 \pm 16.3$; vehicle, $137.8 \pm$ 13.0; and control, $118.8 \pm 12.1 \mathrm{U} / \mathrm{L}$ ) and creatinine (zinc,
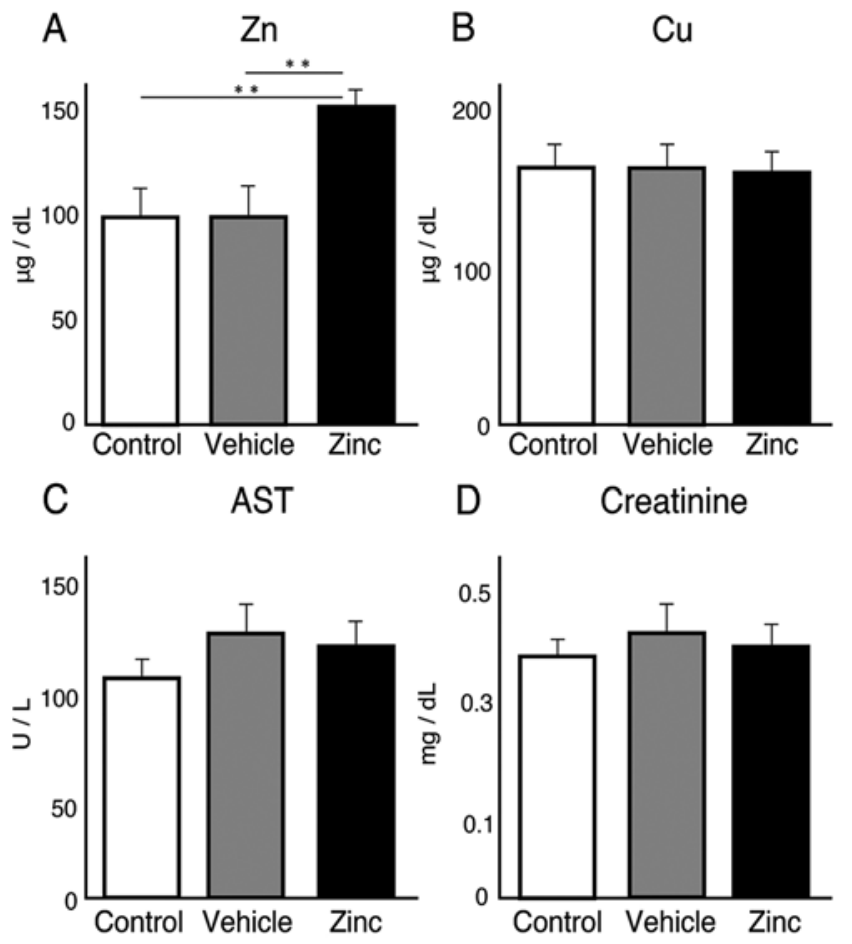

FIG. 2. Serum concentration data for zinc ( $\mathrm{Zn} ; \mathbf{A})$, copper (Cu; B), AST (C), and creatinine (D) in rats 4 weeks after aneurysm induction and in control rats were prepared for evaluation. Data were statistically analyzed using the Kruskal-Wallis test. Results are presented as means \pm SDs for $n=8$ per group, ${ }^{* *} p<0.01$. The total number of animals used was 9 in each group.

$0.482 \pm 0.03$; vehicle, $0.403 \pm 0.02$; and control, $0.383 \pm$ $0.02 \mathrm{U} / \mathrm{L}$ ), which reflect adverse conditions in the liver and kidney, respectively, also did not differ significantly between the 3 groups (Fig. 2C and D).

To determine whether zinc supplementation affects the progression of IAs, we evaluated the morphological and pathological changes in IAs and analyzed wall thickness ratio and aneurysm size. EvG staining demonstrated that administration of zinc prevents the growth of IAs (Fig. $3 \mathrm{~A})$. Accordingly, the aneurysm size in the zinc-administered group was significantly smaller than the size in the vehicle group (zinc, $37.1 \pm 5.4$; vehicle, $80.4 \pm 7.8 \mu \mathrm{m}$ ), whereas the wall thickness ratio was lower in the vehicle group (zinc, $0.59 \pm 0.05$; vehicle, $0.30 \pm 0.04$, p $<0.01$ for the 2 parameters; Fig. 3B). The mortality of surgery was less than $20 \%$. Every rat, excluding those in the control group, developed IAs in the OA-ACA bifurcation. However, in this rat model the development of IAs in the posterior circulation was unlikely. All of the internal elastic laminae of the IAs were disrupted.

\section{Zinc Supplementation Prevented IA by Induced A20}

Immunohistochemical analysis revealed that the expression of A20 in IA walls was increased in the zincadministered group, whereas A20 was barely detectable in the vehicle-administered group (Fig. 4A). By contrast, the vehicle group showed robust accumulation of Iba-1positive macrophages in the IA walls compared with the 

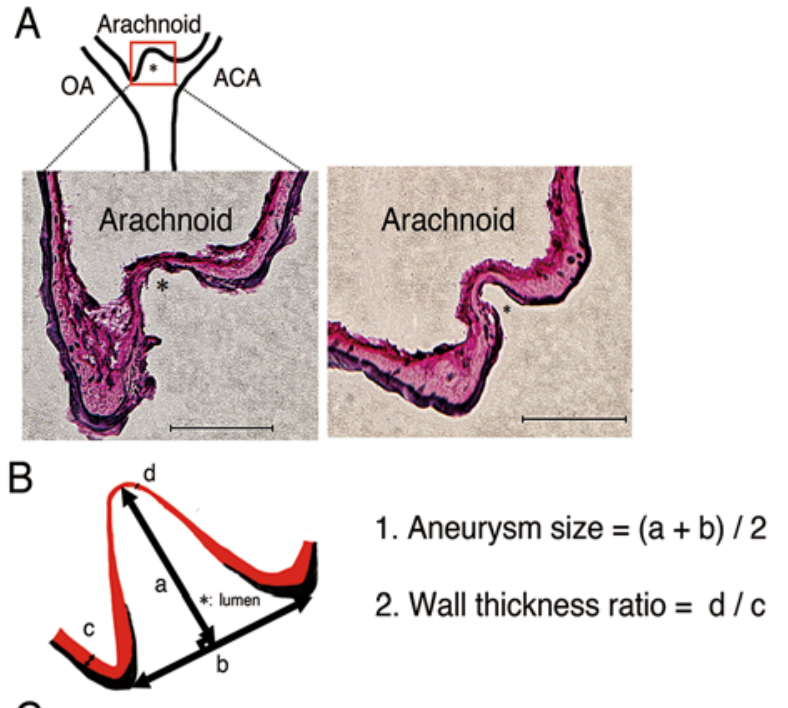

C

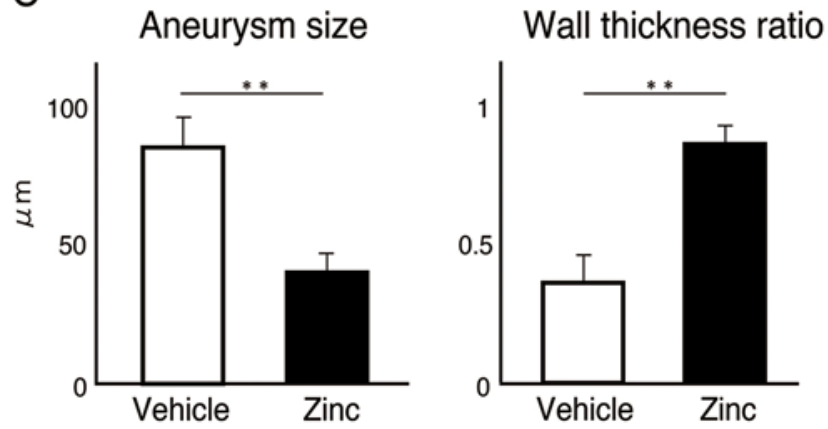

FIG. 3. Administration of zinc prevents the growth of IAs. A: Examples of IAs in the 2 groups. Representative images of IA walls using EvG staining in the group treated with vehicle (left) or zinc (right). Asterisks indicate the luminal sides of IAs. Bars $=50 \mu \mathrm{m}$. B: Schematic representation of the morphological assessment of IAs. The red heavy line represents the aneurysmal wall and the black line represents the internal elastic lamina. C: Morphological assessments were performed with 2 parameters (aneurysm size and wall thickness ratio). Data were statistically analyzed using the Mann-Whitney U-test. Results are presented as means \pm SDs for $n=8$ per group, ${ }^{* *} p<0.01$. The total numbers of animals used were 16 in the vehicle and 17 in the zinc-administered group. Figure is available in color online only.

zinc-administered group (Fig. 4B), meaning that zinc supplementation decreased the inflammatory burden in the blood vessel walls.

Consistently, Western blotting revealed that A20 expression was lower in the vehicle group than in the control group, whereas administration of zinc significantly increased the level of A20 above that in the control group ( $p<0.01$; Fig. 5A). Together, these results demonstrate that administration of zinc augmented A20 expression in IAs.

\section{Zinc Mitigates NF-KB Activation}

Zinc is a key negative regulator of inflammation. Consistent with this characteristic, Western blotting revealed that the levels of the phosphorylated NF- $\kappa \mathrm{B}$ p65 subunit (p-p65), which represents the activated form of NF- $\kappa \mathrm{B},{ }^{14}$, were higher in the vehicle group than in the zinc-administered group ( $p<0.01$; Fig. 4B), whereas the total levels of the NF-кB p65 subunit did not differ significantly between groups (Fig. 5C). In addition, compared with the vehicle group, the levels of phosphorylated IкB $\alpha(\mathrm{p}-\mathrm{I} \kappa \mathrm{B} \alpha)$ decreased in the zinc-administered group, which led to the prevention of I $\mathrm{KB} \alpha$ degradation in IA walls $(\mathrm{p}<0.01$ for both groups; Fig. 5D and E). These results demonstrate that zinc supplementation inhibited NF- $\mathrm{KB}$ activation, thereby downregulating inflammation in IAs.

\section{Discussion}

The results of this study demonstrated that administration of zinc inhibited the growth of experimental IAs and decreased infiltration of inflammatory cells. Furthermore, zinc supplementation induced A20 expression, which possibly resulted in mitigation of the inflammatory NF- $\mathrm{KB}$ signaling pathway in the vessel walls. It is possible that these findings will lead to new strategies for treating IAs.

IAs are relatively common, and IA rupture results in catastrophic SAH, which is associated with excessive risks of morbidity and mortality. ${ }^{2}$ Surgical clipping or endovascular coiling can be offered to patients with unruptured IAs with the goal of preventing aneurysmal rupture; ${ }^{4}$ however, these techniques are highly invasive. Therefore, in order to develop effective medical treatments for unruptured IAs, the mechanisms underlying the formation and the growth of IAs must be clarified. Because the NF- $\kappa \mathrm{B}$ signaling pathway, a central regulator of chronic inflammation, is critically associated with IA formation, ${ }^{3}$ in this study we focused on zinc, a crucial antioxidant as well as an antiinflammatory factor, ${ }^{10}$ in terms of the prevention of experimental IA formation and growth.

Regarding antiinflammatory actions, previous studies indicated that zinc inhibits NF- $\kappa \mathrm{B}$ activation via inducing the $\mathrm{ZnF}$ protein A20. ${ }^{6} \mathrm{~A} 20$ is considered to be an antiinflammatory molecule, ${ }^{7}$ containing 2 ubiquitin-editing domains, an N-terminal deubiquitinating (DUB) domain, and $7 \mathrm{C}$-terminal $\mathrm{ZnF}$ motifs. Together, these domains comprehensively regulate ubiquitin-dependent signals and consequently restrict the interactions of several proteins involved in the NF- $\mathrm{KB}$ pathway. ${ }^{8}$ In fact, most cells exhibit low levels of A20 under normal circumstances. Notably, A20 expression is regulated, at least in part, by NF- $\kappa$ B signaling: upon inflammatory stimulation, A20 is rapidly produced in cells as a feedback mechanism for mitigating the further activation of the NF- $\kappa \mathrm{B}$ pathway. ${ }^{5,10}$ In addition, regulation of the inflammatory response mediated by proinflammatory cytokines is largely under the control of zinc..$^{15}$ Zinc supplementation leads to suppression of inflammatory cytokines via upregulating A20 expression, thereby inhibiting induction of NF- $\mathrm{BB}$ activation. ${ }^{6}$

In this study, we observed higher A20 expression in the zinc-administered than in the vehicle-administered group. Accordingly, the activation of the NF- $\mathrm{BB}$ pathway in the vessel walls, represented by the phosphorylation levels of the p65 subunit and IкB $\alpha$, was markedly suppressed by zinc supplementation. Indeed, zinc supplementation prevents the development of abdominal aortic aneurysms (AAAs) in rats by induction of the expression of A20 in vascular smooth muscle cells and subsequent inhibition of NF- $\kappa B$ signaling. ${ }^{13}$ Consistent with the previous report, in 


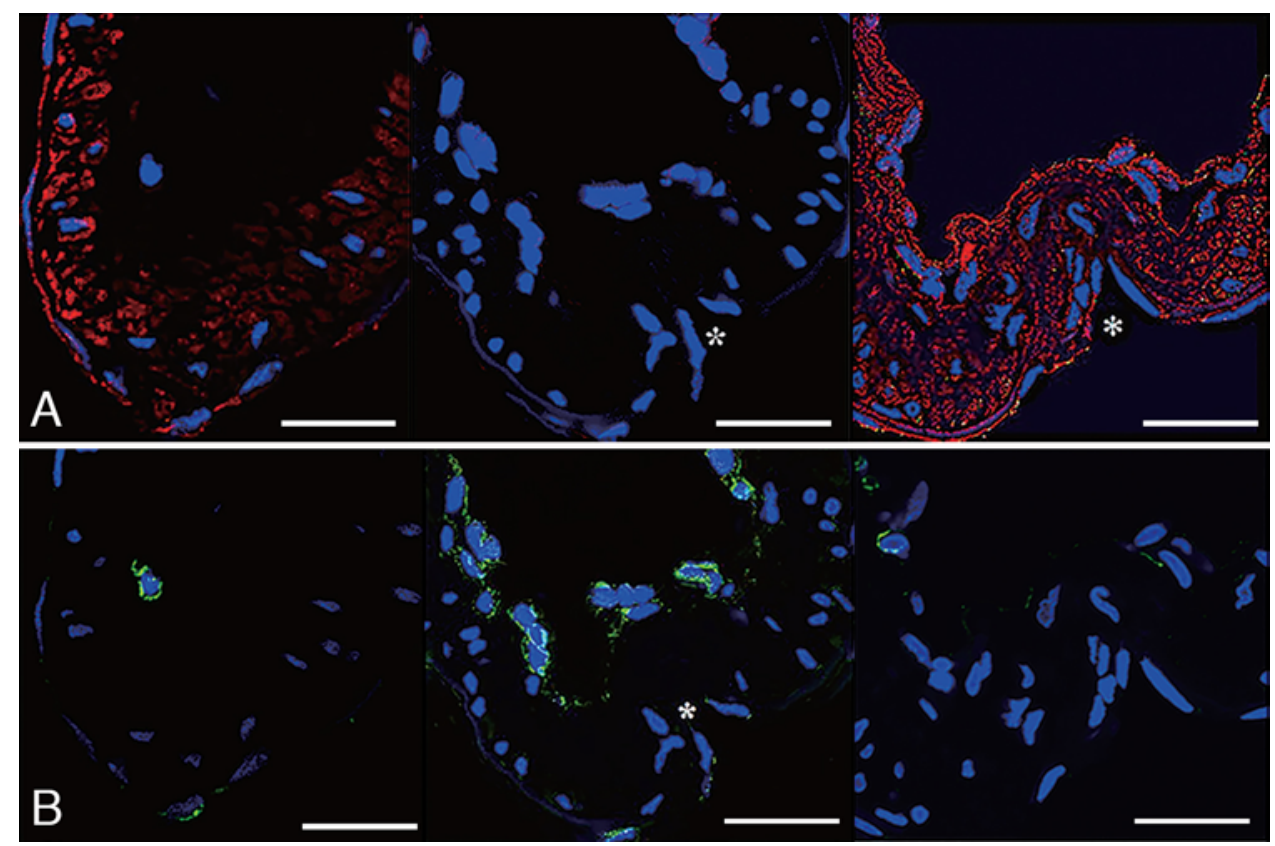

FIG. 4. Treatment with zinc increases $A 20$ expression and suppresses macrophage infiltration in IA walls. Immunohistochemical staining of A20 (red, A) or lba-1-positive macrophages (green, B) with DAPI nuclear staining (blue) in the control (left), vehicle (center), and zinc-treated group (right). Asterisks indicate the luminal sides of IAs. Bar $=20 \mu \mathrm{m}$. The total numbers of animals used were 15 in the control group, 16 in the vehicle group, and 16 in the zinc-administered group. Figure is available in color online only.

this study, IA size and area were significantly smaller in the zinc-administered group, indicating that zinc supplementation may lead to inactivation of the NF- $\mathrm{KB}$ pathway, thereby suppressing the growth of IAs.

Our study indicated that in experimental IAs, activation

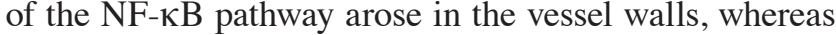
the protein levels of A20 markedly decreased. This finding appeared inconsistent with the cellular response to inflammatory cytokines that can induce A20 expression; 5,10 however, in the experimental AAA model, similarly to our results, expression of A20 protein in aneurysmal lesions is almost undetectable in the vehicle-treated group. ${ }^{13} \mathrm{Al}-$ though the detailed mechanisms remain unknown, A20 expression is regulated at the transcriptional as well as the posttranscriptional level, and posttranscriptional modifications of A20 may control its activity. ${ }^{16}$ It is possible that A20 is either suppressed or degraded in inflammatory settings during aneurysmal formation.

Recently, the citizens of many developed countries have been decreasing their zinc intake by adopting Western diets. Older adults are at particular risk of zinc deficiency because of poor diet, age-related declines in absorption, and urinary overexcretion. ${ }^{17}$ Manifestations of zinc deficiency in humans include dermatitis, delayed wound healing, and reduced levels of serum testosterone, which plays a role in antiinflammation. ${ }^{18}$ No stored pool exists in the body to protect against inadequate zinc intake, and therefore a regular dietary supply is necessary. ${ }^{17}$ Both unruptured and ruptured IAs become more prevalent with age. ${ }^{19}$ So far, no convincing evidence has been provided to support the association of zinc deficiency with the prevalence of SAH or IA; however, our findings may suggest that adequate zinc supplementation would be beneficial, particularly in elderly patients with unruptured IAs, to prevent the enlargement and subsequent rupture of these IAs.

\section{Study Limitations}

This study has several limitations. First, we demonstrated that zinc administration could induce A20 expression and suppress NF- $\kappa \mathrm{B}$ activation in experimental rat IAs; however, we did not test the effects of zinc on human IAs. Therefore, future studies should seek to determine whether zinc can modulate the NF- $\kappa \mathrm{B}$ pathway by inducing A20 in human IAs. Second, we used male rats for the present study to exclude the effect of female hormones. In females, the presence of female hormones such as estrogen leads to a decreased tendency toward aneurysm formation and rupture..$^{20,21}$ Because zinc supplementation increases serum levels of estrogen and progesterone in ovariectomized rats ${ }^{22}$ the preventive effect of zinc supplementation on IA growth may be stronger in female than in male rats. Third, in the present study we used $0.12 \%$ $\beta$-aminopropionitrile (BAPN), which inhibits the crosslinking between elastin and collagen. ${ }^{23}$ Although there is no evidence so far that BAPN affects the inflammatory cascades involved in IA development, the possibility that BAPN had an influence on the results cannot be ruled out. Fourth, we did not demonstrate the causality between the increase in A20 expression and inhibition of the NF- $\mathrm{B}$ pathway in this model, although the molecular mechanism by which A20 regulates NF- $\mathrm{KB}$ activation is well characterized in other settings. It would have been more suitable to use A20-knockout mice in this investigation; however, A20-knockout mice are cachectic and show premature death because of excessive multiple-organ inflammation. ${ }^{24}$ 


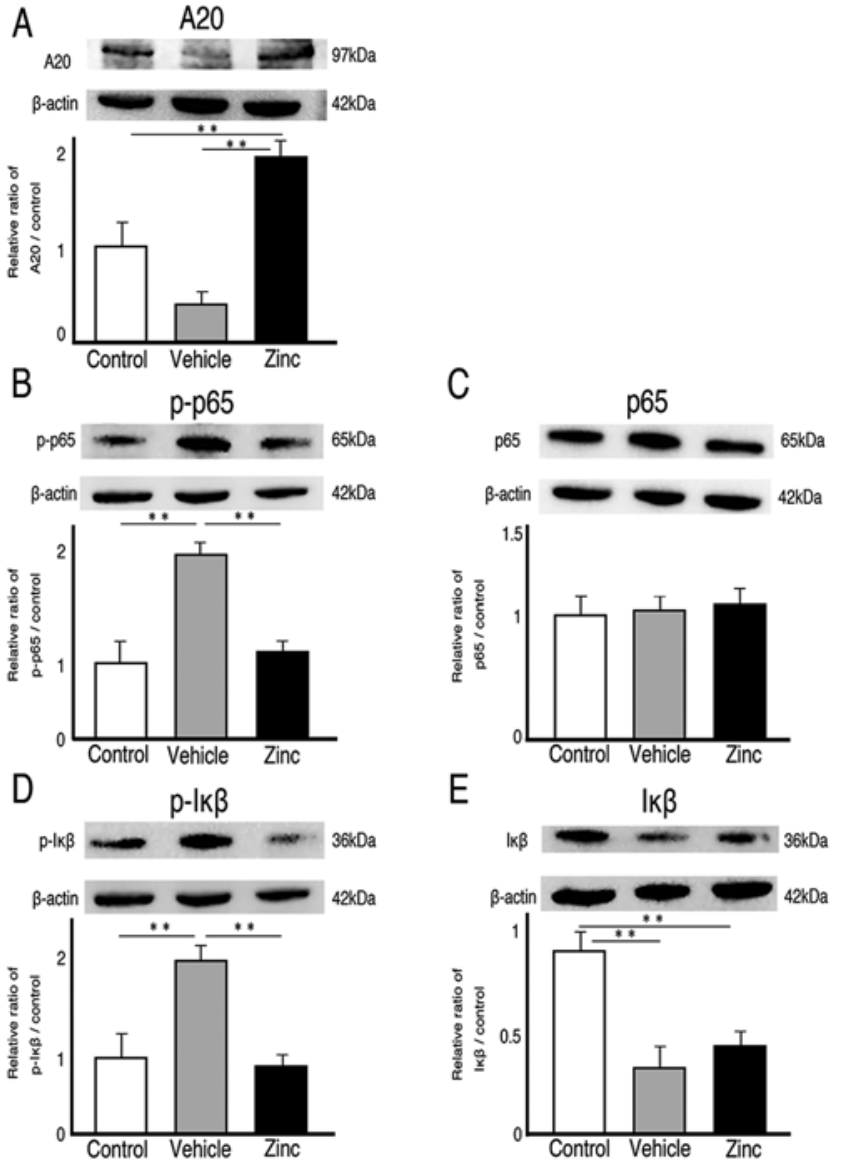

FIG. 5. Zinc supplementation augments A20 protein expression and subsequently inhibits the NF-KB signaling pathway. Samples obtained from the circle of Willis were harvested 4 weeks after aneurysm induction and subjected to Western blot analyses using antibodies specific for A20 (A), phospho-NF-кB p65 (p-p65) (B), NF-кB p65 (p65, total) (C),

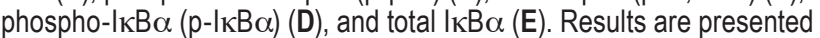
as means \pm SDs for $n=6$ per group. The total numbers of animals used were 21 in the control group, 19 in the vehicle group, and 18 in the zincadministered group.

\section{Conclusions}

Zinc supplementation induced expression of A20, a potent antiinflammatory molecule, and decreased activation of the NF- $\kappa \mathrm{B}$ pathway in the vessel walls in experimentally induced IAs in rats. Consequently, zinc replenishment prevents the growth of IAs. Impaired zinc homeostasis may cause multiple chronic diseases and is relatively common in developed countries, particularly in the elderly. Although further studies in human IAs are necessary, our findings indicate that zinc administration may be a potent and useful therapy to prevent the enlargement and subsequent rupture of IAs.

\section{Acknowledgments}

We thank Dr. Tomohiro Aoki (National Cerebral and Cardiovascular Center Research Institute, Suita, Japan) for lending his expertise in generating experimental intracranial aneurysms in rats. This work was supported in part by Japan Society for the
Promotion of Science (JSPS) KAKENHI grants 26460338 and 17K08592 (to M.M.) and 15H04952 (to H.K.).

\section{References}

1. Aoki T, Kataoka H, Shimamura M, et al. NF-kappaB is a key mediator of cerebral aneurysm formation. Circulation. 2007;116(24):2830-2840.

2. Feigin VL, Findlay M. Advances in subarachnoid hemorrhage. Stroke. 2006;37(2):305-308.

3. Kataoka H. Molecular mechanisms of the formation and progression of intracranial aneurysms. Neurol Med Chir (Tokyo). 2015;55(3):214-229.

4. Ikedo T, Minami M, Kataoka H, et al. Dipeptidyl peptidase-4 inhibitor anagliptin prevents intracranial aneurysm growth by suppressing macrophage infiltration and activation. $\mathrm{J} \mathrm{Am}$ Heart Assoc. 2017;6(6):e004777.

5. He KL, Ting AT. A20 inhibits tumor necrosis factor (TNF) alpha-induced apoptosis by disrupting recruitment of TRADD and RIP to the TNF receptor 1 complex in Jurkat T cells. Mol Cell Biol. 2002;22(17):6034-6045.

6. Prasad AS, Bao B, Beck FW, et al. Antioxidant effect of zinc in humans. Free Radic Biol Med. 2004;37(8):1182-1190.

7. Bosanac I, Wertz IE, Pan B, et al. Ubiquitin binding to A20 $\mathrm{ZnF} 4$ is required for modulation of NF- $\mathrm{\kappa B}$ signaling. Mol Cell. 2010;40(4):548-557.

8. Tokunaga F, Nishimasu H, Ishitani R, et al. Specific recognition of linear polyubiquitin by A20 zinc finger 7 is involved in NF- $\kappa \mathrm{B}$ regulation. EMBO J. 2012;31(19):3856-3870.

9. Aoki T, Nishimura M, Kataoka H, et al. Reactive oxygen species modulate growth of cerebral aneurysms: a study using the free radical scavenger edaravone and $\mathrm{p} 47 \mathrm{phox}^{-/-}$mice. Lab Invest. 2009;89(7):730-741.

10. Prasad AS. Clinical, immunological, anti-inflammatory and antioxidant roles of zinc. Exp Gerontol. 2008;43(5):370-377.

11. Nagata I, Handa H, Hashimoto N. Experimentally induced cerebral aneurysms in rats: part IV-cerebral angiography. Surg Neurol. 1979;12(5):419-424.

12. Ozturk A, Baltaci AK, Mogulkoc R, Oztekin E. Zinc prevention of electromagnetically induced damage to rat testicle and kidney tissues. Biol Trace Elem Res. 2003;96(1-3):247-254.

13. Yan YW, Fan J, Bai SL, et al. Zinc prevents abdominal aortic aneurysm formation by induction of A20-mediated suppression of NF-кB pathway. PLoS One. 2016;11(2):e0148536.

14. Schneider A, Martin-Villalba A, Weih F, et al. NF-kappaB is activated and promotes cell death in focal cerebral ischemia. Nat Med. 1999;5(5):554-559.

15. Tokunaga F, Iwai K. LUBAC, a novel ubiquitin ligase for linear ubiquitination, is crucial for inflammation and immune responses. Microbes Infect. 2012;14(7-8):563-572.

16. Ma A, Malynn BA. A20: linking a complex regulator of ubiquitylation to immunity and human disease. Nat Rev Immunol. 2012;12(11):774-785.

17. Lowe NM, Fekete K, Decsi T. Methods of assessment of zinc status in humans: a systematic review. Am J Clin Nutr. 2009;89(6):2040S-2051S

18. Vasto S, Mocchegiani E, Malavolta M, et al. Zinc and inflammatory/immune response in aging. Ann N Y Acad Sci. 2007;1100:111-122.

19. Hishikawa T, Date I, Tokunaga K, et al. Risk of rupture of unruptured cerebral aneurysms in elderly patients. Neurology. 2015;85(21):1879-1885.

20. Kubo Y, Koji T, Kashimura H, et al. Female sex as a risk factor for the growth of asymptomatic unruptured cerebral saccular aneurysms in elderly patients. J Neurosurg. 2014;121(3):599-604.

21. Tada Y, Makino H, Furukawa H, et al. Roles of estrogen in the formation of intracranial aneurysms in ovariectomized female mice. Neurosurgery. 2014;75(6):690-695. 
22. Sunar F, Baltaci AK, Ergene N, Mogulkoc R: Zinc deficiency and supplementation in ovariectomized rats: their effect on serum estrogen and progesterone levels and their relation to calcium and phosphorus. Pak J Pharm Sci. 2009;22:150-154.

23. Aoki T, Kataoka H, Ishibashi R, et al. Reduced collagen biosynthesis is the hallmark of cerebral aneurysm: contribution of interleukin-1beta and nuclear factor-kappaB. Arterioscler Thromb Vasc Biol. 2009;29(7):1080-1086.

24. Lee EG, Boone DL, Chai S, et al. Failure to regulate TNF-induced NF-kappaB and cell death responses in A20-deficient mice. Science. 2000;289(5488):2350-2354.

\section{Disclosures}

The authors report no conflict of interest concerning the materials or methods used in this study or the findings specified in this paper.

\section{Author Contributions}

Conception and design: Minami, Kataoka. Acquisition of data: Hayashi, Ikedo, Miyata, Shimizu, Nagata, Yang, Yamamoto.

Analysis and interpretation of data: Minami, Hayashi, Kataoka, Ikedo, Miyata, Shimizu, Nagata, Yang, Yamamoto. Drafting the article: Hayashi. Critically revising the article: Minami, Kataoka, Yokode, Miyamoto. Reviewed submitted version of manuscript: Minami, Hayashi, Kataoka, Yokode, Miyamoto. Approved the final version of the manuscript on behalf of all authors: Minami. Statistical analysis: Hayashi. Administrative/technical/material support: Minami, Kataoka, Yokode, Miyamoto. Study supervision: Minami, Kataoka, Yokode, Miyamoto.

\section{Correspondence}

Manabu Minami: Kyoto University Graduate School of Medicine, Kyoto, Japan.mminami@kuhp.kyoto-u.ac.jp. 